\title{
Performance Analysis of Correlated Multiple Antenna Spectrum Sensing Cognitive Radio
}

\author{
Aamir Z. Shaikh \\ Department of Telecommunications Engineering, \\ NED University of Engineering \& Technology, \\ Karachi. Pakistan.
}

\author{
Talat Altaf \\ $\mathrm{PhD}$, Department of Electrical Engineering, \\ NED University of Engineering \& Technology, \\ Karachi. Pakistan.
}

\begin{abstract}
We derive probability of detection $P_{d}$ and false alarm $P_{f}$ for spectrum sensing cognitive devices, employing correlated multiple antenna elements using linear test statistic. Detection performance of such sensors is severely degraded due to the correlation among antennas, in addition to that fading channel conditions may further deteriorate the performance. We propose a simple hard decision fusion strategy at the secondary Base Station to improve the performance by exploiting collaborative gain. Region of Convergence (ROC) is also evaluated under OR based fusion strategy. Numerical results certify the proposed proposal.
\end{abstract}

\section{Keywords}

correlated multiple antenna energy detector, linear statistic, cognitive radio, cooperation, collaboration gain, hard decision strategy.

\section{INTRODUCTION}

Cognitive Radio is a revolutionary concept that promises to resolve spectrum scarcity problem by exploiting unused spectrum spaces (a.k.a. Holes) in an opportunistic fashion. These radios apply intelligent signal processing algorithms to detect unused spectrum bands available at a specific time, frequency and geographic area. And use those identified bands for their communication subject to protection of Primary users from harmful interference as per Federal Communications Commission (FCC) rules [1].

Spectrum Sensing is the essential part of any intelligent cognitive device. Sensing is important both for detection of spectrum holes to exploit in secondary manner and to protect primary users from harmful interference of secondary users. Spectrum Sensing can be implemented through many techniques such as Energy Detection, Cyclostationary Feature Detection, Matched Filter Detection, Eigen value Detection and Waveform Detection. (A comprehensive review of sensing techniques is available in [2]).

Energy Detection technique, in comparison to other sensing techniuques is the only algorithm which requires no prior information about primary user. Energy of received signal samples is computed and compared with a pre-set threshold (which depends on noise level). In case, the received signal energy is larger than a fixed threshold, detector decides the band as occupied and in other case band is tagged as white space (unused spectrum band). Energy detector, thus, distinguishes between the occupied and empty bands (White Spaces) on the basis of received energy and cannot therefore differentiate between various types of signals, interference, primary signal and noise [2]. Thus, in low SNR region, useful signal and arbitrary noise become indistinguishable [3], resulting in an increase of error probabilities ie Probability of false alarm and missed-detection.

One possible way to improve Region of Convergence (ROC) performance of energy detector in case of low SNR regime is by using multiple antenna cognitive radio which exploits spatial diversity [4-12]. In [4] authors derive detection performance of multiple correlated antennas and verify that the amount of correlation among antennas is directly proportional to degradation of performance. In [5], authors derive multi antenna based optimal and Generalized Likelihood ratio detectors (GLRD). GLRDs are preferred in case of unknown channel parameters including channel gains, noise and PU signal variance. It is also shown that GLRD performs better than Energy detector and almost identical to optimal detector under noise variance mismatch. In [6], authors derive the performance of multiple antenna detectors by combining the sensing results using soft decision algorithms including Maximal ratio combining and Selection processing. Hence it is concluded that even under low to moderate SNRs detection performance is on higher side. Furthermore, it is shown that MRC based detector gives an upper bound on detection performance because it uses channel information and optimum coherent combining. In [7] authors derive performance of improved energy detector based on multiple antenna strategy under Cooperation scenario. It is verified that the detection performance can be improved with significant reliability even at very low SNR levels with very low interference levels to Primary User. In [8] authors study performance of multiple antenna energy detectors under equally correlated, exponentially correlated and linear array in Nakagami-m fading channels. The sensing results are combined through Square Law Combining technique. The results are derived using Moment Generating Function (MGF) based approach. In [9] author analyzes the detection performance of multiple antenna based Cognitive Radio using autocorrelation of received samples. Furthermore, variance at different antenna is assumed as different without the knowledge of their values.

Furthermore, blind detectors are proposed in literature to improve detection performance of multiple antenna based sensing algorithms in case of weak Signal to Noise Ratio and noise uncertain conditions. These schemes exploit other parameters of received signals (which do not require noise floor for threshold setting as in case of Energy Detectors) for detection of spectral holes. These parameters include characteristic function [10] which is evaluated by taking Fourier transform of probability density function (PDF) of received samples, Polarity coincidence Array (PCA) [11] and 
dimension estimation based Minimum Description Length (MDL) [12]. As these detectors do not depend on the noise floor so they perform significantly better than the energy detectors especially in case of weak received signals.

We derive detection performance of correlated multiple antenna based energy detectors. The difference between our work and the [4] is that we measure performance using linear test statistic which is computationally efficient method in comparison to quadratic detectors and partial test statistic method employed in [4]. Furthermore, in [4], authors verify the performance degradation of multiple antenna detectors under correlated antennas but no method is proposed to improve performance in such scenario. We employ a simple hard decision fusion strategy at secondary BS to improve detection performance by exploiting collaborative gains. The proposed architecture is shown in Figure 1.

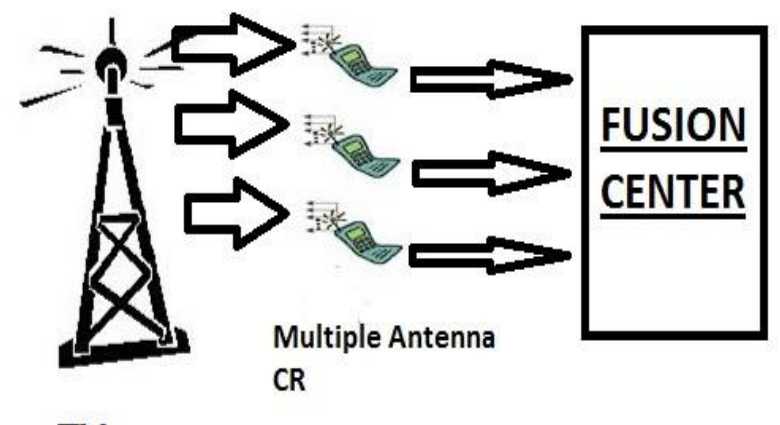

TV

\section{Transmitter}

Figure 1. Proposed architecture for energy detector with correlated multiple antennas.

In Section 2 we present System Model. In section 3 Hypothesis testing rule and Performance Analysis is derived for correlated multiple antenna sensors. In section 4 Numerical Results of the proposed technique is presented. We conclude the paper in section 5.

\section{SYSTEM MODEL}

Spectrum sensing can be formulated as a simple binary hypothesis testing rule:

$$
y(n)=\left\{\begin{array}{cc}
\mathbf{w}_{\mathbf{i}}(\mathbf{n}) & ; H_{A} \\
\mathbf{h}_{\mathbf{i}}(\mathbf{n}) \mathbf{x}(\mathbf{n})+\mathbf{w}_{\mathbf{i}}(\mathbf{n}) & ; H_{B}
\end{array}\right.
$$

For $n=0,1,2, \ldots, k$ shows the number of samples, $\mathbf{w}_{\mathbf{i}}(\mathbf{n})$ represents samples of additive White Gaussian noise, $w_{i}(n) \sim \mathbb{N}\left(0 . P_{n}\right)$ and $\mathbf{h}_{\mathbf{i}}(\mathbf{n})$ shows Rayleigh channel fading coefficients, $h(n) \sim \mathbb{N}\left(0, P_{n}\right)$ and $\mathbf{x}(\mathbf{n})$ shows the samples of Primary User signal. Under hypothesis $H_{A}$, samples of received signal consist of noise only indicating absence of Primary User, whereas under $H_{B}$, samples of received signal constitute both primary user signal and noise, indicating the presence of a Legitimate/Licensed user.

y can be defined as a multivariate Gaussian random variable with following distribution:

$$
y \sim\left\{\begin{array}{l}
\mathbb{N}\left(\mu_{A}, \sum_{A}\right) ; H_{A} \\
\mathbb{N}\left(\mu_{B}, \sum_{B}\right) ; H_{B}
\end{array}\right.
$$

Where $\mu_{A}$ and $\mu_{B} \in \mathbb{N}^{K \times 1}$ represent mean of received signal while $\sum_{\mathbf{A}}$ and $\sum_{\mathbf{B}} \in \mathbb{N}^{K \times K}$ the Covariance matrices of $\mathbf{y}$ under hypothesis $H_{A}$ and $H_{B}$ respectively.

Mean and Covariance Matrices can be defined as:

$$
\begin{array}{cl}
\boldsymbol{\mu}_{\mathbf{A}}=P_{n} \times \mathbf{1}, & \boldsymbol{\mu}_{\mathbf{B}}=\left(P P_{h}+P_{n}\right) \times \mathbf{1} \\
\Sigma_{\mathbf{A}}=\frac{P_{n}^{2}}{m} \mathbf{I}_{\mathbf{K}}, & \Sigma_{\mathbf{B}}=\frac{P_{n}^{2}}{m} \mathbf{I}_{\mathbf{K}}+\frac{2 P P_{h} P_{n}}{m} \boldsymbol{\Lambda}
\end{array}
$$

Where $\mathbf{I}_{\mathbf{K}}$ is a $K \times K$ Identity matrix and $\boldsymbol{\Lambda}$ is a $K \times K$ covariance matrix. The sensing radio consists of $K$ correlated antennas, each antenna collects $K$ number of samples. $\Lambda$ can be defined [13] as:

$$
\boldsymbol{\Lambda}_{p q}= \begin{cases}\rho^{p-q} & , p \leq j \\ \Lambda_{q p}^{*} & , p>q\end{cases}
$$

For $p, q=1,2, \ldots, K$ and $0 \leq \rho \leq 1$, where $\rho$ is an antenna correlation coefficient defined [13] as:

$$
\rho=\exp \left[-23 \Omega^{2}\left(\frac{s}{\lambda_{c}}\right)^{2}\right]
$$

$\Omega$ is angular speed, $\lambda_{c}$ represents wavelength of the received signal while $\mathrm{s}$ being the space between two adjacent elements of multiple antenna. In [4] it is shown that under above configuration, $\Lambda$ is a symmetric Toeplitz matrix [4]

\section{HYPOTHESIS TESTING \& PERFORMANCE ANALYSIS}

The Probability distribution under each hypothesis can be formulated as:

$$
\begin{gathered}
f_{A}(\mathbf{y})=\frac{1}{(2 \pi)^{\frac{k}{2}}\left|\Sigma_{A}\right|^{\frac{1}{2}}} \exp \left\{-\frac{1}{2}\left(\mathbf{y}-\boldsymbol{\mu}_{\mathbf{A}}\right)^{T} \Sigma_{A}^{-1}\left(\mathbf{y}-\boldsymbol{\mu}_{\mathbf{A}}\right)\right\} \\
f_{B}(\mathbf{y})=\frac{1}{(2 \pi)^{\frac{k}{2}}\left|\Sigma_{B}\right|^{\frac{1}{2}}} \exp \left\{-\frac{1}{2}\left(\mathbf{y}-\boldsymbol{\mu}_{\mathbf{B}}\right)^{T} \Sigma_{B}^{-1}\left(\mathbf{y}-\boldsymbol{\mu}_{\mathbf{B}}\right)\right\}
\end{gathered}
$$

Considering Neyman-Pearson Rule, The Likelihood Ratio Test can be formulated from equation (7) and equation (8):

$$
\begin{aligned}
\Upsilon(\mathbf{y})= & \frac{\left(\mathbf{x} ; H_{B}\right)}{\left(\mathbf{x} ; H_{A}\right)} \\
= & \frac{1}{(2 \pi)^{\frac{k}{2}}\left|\Sigma_{B}\right|^{\frac{1}{2}}} \exp \left\{-\frac{1}{2}\left(\mathbf{y}-\boldsymbol{\mu}_{\mathbf{B}}\right)^{T} \Sigma_{B}^{-1}\left(\mathbf{y}-\boldsymbol{\mu}_{\mathbf{B}}\right)\right\} \\
& \frac{1}{(2 \pi)^{\frac{k}{2}}\left|\sum_{A}\right|^{\frac{1}{2}}} \exp \left\{-\frac{1}{2}\left(\mathbf{y}-\boldsymbol{\mu}_{\mathbf{A}}\right)^{T} \Sigma_{A}^{-1}\left(\mathbf{y}-\boldsymbol{\mu}_{\mathbf{A}}\right)\right\}
\end{aligned}
$$

Assume $\Sigma_{A}=\sum_{B}=\sum$ by considering the fact that unwanted noise present during $H_{A}$ makes the covariance under both hypothesis equal. By doing so, the quadratic test statistic is transformed into linear test statistic, thus making the test statistic computationally efficient. Taking log on both sides, equation (9) becomes

$$
\begin{aligned}
& \ln \Upsilon(\mathbf{y})=\frac{1}{2} \ln \frac{\left|\Sigma_{\mathbf{A}}\right|}{\left|\Sigma_{\mathbf{B}}\right|}+\mathbf{y}_{\mathrm{A}}^{\mathrm{T}} \Sigma_{\mathrm{A}}{ }^{-1} \mathbf{y}_{\mathrm{A}}-\mathbf{y}_{\mathrm{B}}^{\mathrm{T}} \Sigma_{\mathrm{B}}{ }^{-1} \mathbf{y}_{\mathbf{B}} \\
& \mathbf{y}_{\mathbf{A}}=\mathbf{y}-\boldsymbol{\mu}_{\mathbf{A}} ; \quad \mathbf{y}_{\mathbf{B}}=\mathbf{y}-\boldsymbol{\mu}_{\mathbf{B}}
\end{aligned}
$$

Thus, comparing test statistic with threshold: 


$$
\boldsymbol{\beta}^{\mathbf{T}} \underset{H_{0}}{\stackrel{H_{1}}{>}} \lambda
$$

Which can be formulated as:

$$
\boldsymbol{\beta}^{T}=\left(\boldsymbol{\mu}_{\mathbf{B}}-\boldsymbol{\mu}_{\mathbf{A}}\right)^{T} \boldsymbol{\Sigma}^{-1} ; \quad \lambda=\tau-\boldsymbol{\mu}_{\mathbf{A}}{ }^{\mathrm{T}} \boldsymbol{\Sigma}^{-1} \boldsymbol{\mu}_{\mathbf{A}}+\boldsymbol{\mu}_{\mathbf{B}}{ }^{\mathrm{T}} \boldsymbol{\Sigma}^{-1} \boldsymbol{\mu}_{\mathbf{B}}
$$

Using above assumptions, we derive analytical relations for detection and false alarm probabilities in closed form: $P_{f}=\operatorname{Pr}\left(\right.$ DecideH $_{B} \mid H_{A}$ True $)=\operatorname{Pr}\left(\boldsymbol{\beta}^{\mathbf{T}} \mathbf{y} \succ \lambda \mid H_{A}\right)$

$$
P_{f}=\mathbf{Q}\left(\frac{\lambda-\boldsymbol{\beta}^{\mathbf{T}} \boldsymbol{\mu}_{\mathbf{A}}}{\sqrt{\boldsymbol{\beta}^{\mathbf{T}} \boldsymbol{\Sigma} \boldsymbol{\beta}}}\right)
$$

$P_{d}=\operatorname{Pr}\left(\right.$ Decide $_{B} \mid H_{B}$ True $)=\operatorname{Pr}\left(\beta^{T} y \succ \lambda \mid H_{B}\right)$

$$
P_{d}=\mathbf{Q}\left(\frac{\sqrt{\boldsymbol{\beta}^{\mathrm{T}} \boldsymbol{\Sigma} \boldsymbol{\beta}}\left(P_{f}\right)-\boldsymbol{\beta}^{\mathbf{T}}\left(\boldsymbol{\mu}_{\mathbf{A}}-\boldsymbol{\mu}_{\mathbf{B}}\right)}{\sqrt{\boldsymbol{\beta}^{\mathrm{T}} \Sigma \boldsymbol{\beta}}}\right)
$$

Thus, $\mathrm{P}_{\mathrm{d}}$ can be defined in terms of $\mathrm{P}_{\mathrm{f}}$ as:

$$
P_{d}=\mathbf{Q}\left(\mathbf{Q}^{-1}\left(P_{f}\right)-\sqrt{\boldsymbol{\beta}^{\mathbf{T}} \boldsymbol{\Sigma} \boldsymbol{\beta}}\right)
$$

\section{NUMERICAL RESULTS}

In this section, we present the evaluation of theoretical and analytical propositions presented in the paper. These include both the impact of number of samples on false alarm probability and ROC curves (both single User and fusion center results using OR) for energy sensor with correlated multiple antenna elements under the framework of First IEEE 802.22 standard for Wireless regional Area Network (WRAN). The parameters used for numerical evaluation include following unless otherwise described explicitly: $\underline{P}_{n}=$ $-95.2 \mathrm{dBm}, T=1 \mathrm{mS}, W=6 \mathrm{MHz}, P P_{h}=-114 \mathrm{dBm}, P_{d}=0.9$, $\lambda_{C}=0.5^{\circ}, s=\lambda_{C} / 8$.

Figure 2 illustrates the impact of number of samples on relationship between false alarm probability and received power. Thus, it is obvious that an increase of number of received samples decreases probability of false alarm even on lower received power levels.

Figure 3 shows the impact of number of number of samples on the relation between probability of miss-detection and the received power. When the detector uses 5 samples it completely misses the target till $-105 \mathrm{dBm}$, from where it starts to identify and the probability of miss detection decreases thereof.

Figure 4 compares the benefit of cooperative strategy in comparison to a single sensor with correlated multiple antenna elements. Our proposed strategy improves the Complementary ROC performance significantly.

\section{CONCLUSION}

Detection performance of correlated multiple antenna based cognitive radios is derived using linear test statistic along with its performance evaluation. Furthermore, it is shown that the cooperation among multiple antenna based correlated sensors can be employed to improve overall detection performance significantly.

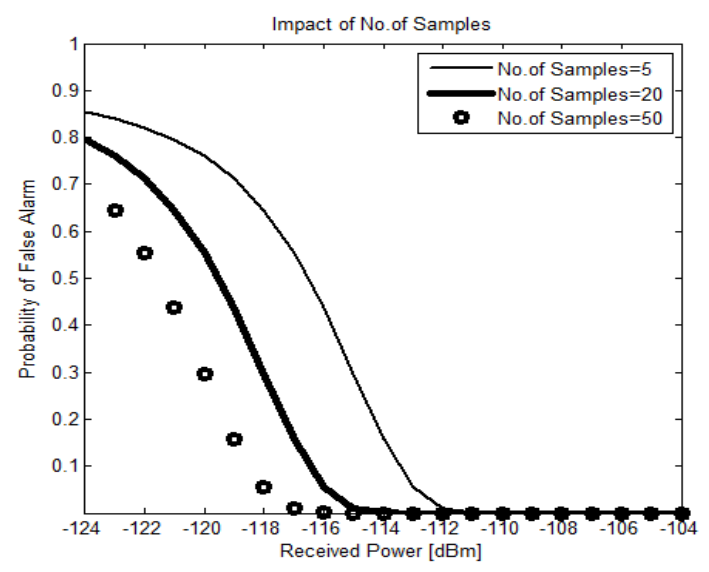

Figure 2. Probability of false alarm and received power

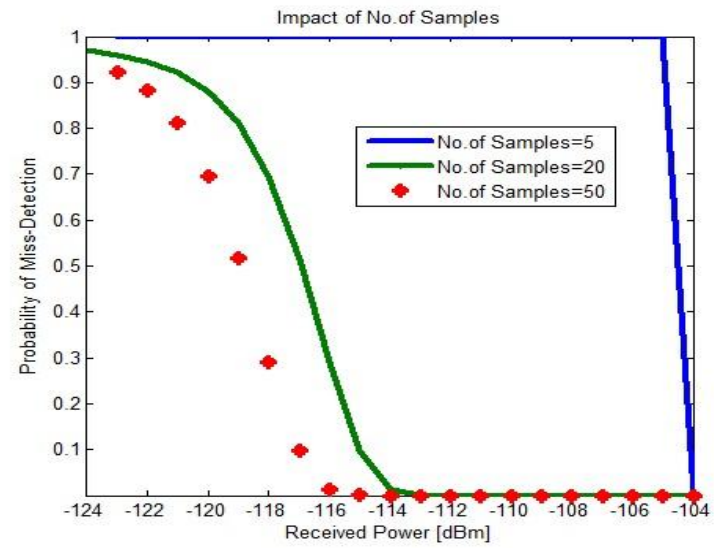

Figure 3. Probability of miss-detection and received power

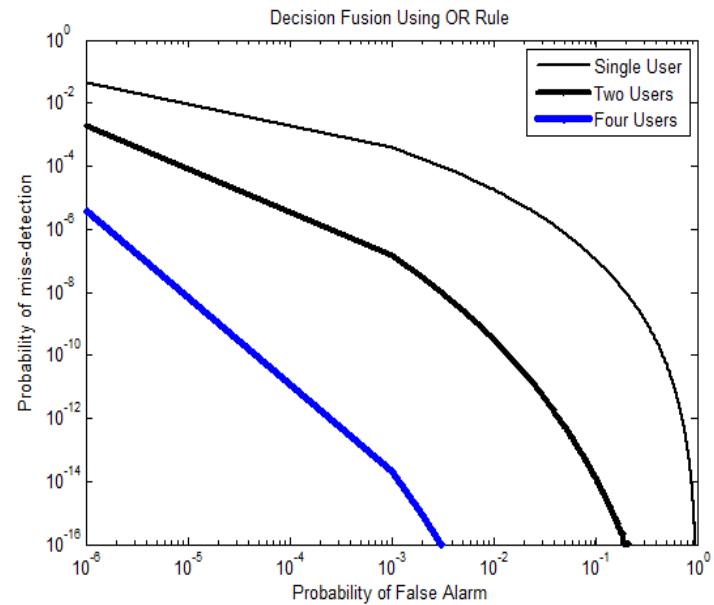

Figure 4. Complementary ROC: Impact of cooperation

\section{ACKNOWLEDGEMENT}

Authors would like to thank Prof. Lakshman Tamil at the University of Texas and Prof. Shoaib Zaidi at NED University of Engineering and Technology for useful discussions and suggestions. A. Z. Shaikh would like to thank Dr. Muhammad Imran Aslam at NED University of Engineering and Technology for his help in preparation of CRC version of this paper. 


\section{REFERENCES}

[1] Haykin, S. 2005. Cognitive radio: brain-empowered wireless communications. Selected Areas in Communications, IEEE Journal on, vol.23, no.2, pp. 201220, Feb. 2005.

[2] Yucek, T.; Arslan, H. 2009. A survey of spectrum sensing algorithms for cognitive radio applications," Communications Surveys \& Tutorials, IEEE , vol.11, no.1, pp.116-130, First Quarter 2009.

[3] Tandra, R.; Sahai, A. 2008. SNR Walls for Signal Detection. IEEE Journal of Selected Topics in Signal Processing,Volume:2,Issue: 1.2008.

[4] Sungtae Kim; Jemin Lee; Hano Wang; Daesik Hong. 2009. Sensing Performance of Energy Detector With Correlated Multiple Antennas," Signal Processing Letters, IEEE, vol.16, no.8, pp.671-674, Aug. 2009

[5] Taherpour, A.; Nasiri-Kenari, M.; Gazor, S. 2010.Multiple antenna spectrum sensing in cognitive radios. Wireless Communications, IEEE Transactions on , vol.9, no.2, pp.814-823, February 2010.

[6] Pandharipande, A.; Linnartz, J.-P.M.G. 2007.Performance Analysis of Primary User Detection in a Multiple Antenna Cognitive Radio. Communications, 2007. ICC '07. IEEE International Conference on , vol., no., pp.6482-6486, 24-28 June 2007.

[7] Singh, A.; Bhatnagar, M.R.; Mallik, R.K. 2012.Cooperative Spectrum Sensing in Multiple Antenna Based Cognitive Radio Network Using an Improved
Energy Detector. Communications Letters, IEEE, vol.16, no.1, pp.64-67, January 2012

[8] Banjade, V.R.S.; Rajatheva, N.; Tellambura, C.2012.Performance Analysis of Energy Detection with Multiple Correlated Antenna Cognitive Radio in Nakagami-m Fading. Communications Letters, IEEE vol.16, no.4, pp.502-505, April 2012

[9] Tugnait, J.K. 2012. On Multiple Antenna Spectrum Sensing Under Noise Variance Uncertainty and Flat Fading. IEEE Transactions on Signal Processing. Volume: $\quad 60$, Issue: 4. 2012 page(s): 1823-1832.

[10] Lei Shen; Haiquan Wang; Wei Zhang; Zhijin Zhao, 2012. Multiple Antennas Assisted Blind Spectrum Sensing in Cognitive Radio Channels. Communications Letters, IEEE, vol.16, no.1, pp.92-94, January 2012.

[11] Wimalajeewa, T.; Varshney, P.K. 2011. PolarityCoincidence-Array Based Spectrum Sensing for Multiple Antenna Cognitive Radios in the Presence of NonGaussian Noise. Wireless Communications, IEEE Transactions on , vol.10, no.7, pp.2362-2371, July 2011.

[12] Zayen, B.; Hayar, A.2012. Dimension estimation based detector for multiple-antenna cognitive radio networks. Telecommunications (ICT), 2012 19th International Conference on , vol., no., pp.1-4, 23-25 April 2012

[13] G. D. Durgin and T.S. Rappaport. 1999.Effects of Multipath Angular Spread on The Spatial CrossCorrelation of Received Voltage Envelopes. IEEE 49th Vehicular Technology Conference, Vol 2, pp.996-1000, May 1999. 